\title{
ON THE INDIRECT DETECTION OF SODIUM IN THE ATMOSPHERE OF THE PLANETARY COMPANION TO HD 209458
}

\author{
J. J. Fortney,${ }^{1}$ D. Sudarsky, ${ }^{2}$ I. Hubeny, ${ }^{3,4}$ C. S. Cooper, ${ }^{1}$ W. B. Hubbard, ${ }^{1}$ \\ A. Burrows, ${ }^{2}$ AND J. I. Lunine ${ }^{1}$ \\ Received 2002 August 13; accepted 2003 January 28
}

\begin{abstract}
Using a self-consistent atmosphere code, we construct a new model of the atmosphere of the transiting extrasolar giant planet HD 209458b to investigate the disparity between the observed strength of the sodium absorption feature at $589 \mathrm{~nm}$ and the predictions of previous models. For the atmospheric temperaturepressure profile we derive, silicate and iron clouds reside at a pressure of several millibars in the planet's atmosphere. These clouds have significant vertical extent and optical depth because of our slant viewing geometry and lead to increased absorption in bands directly adjacent to the sodium line core. Using a nonLTE sodium ionization model that includes photoionization by stellar UV flux, collisional processes with $\mathrm{H}_{2}$, and radiative recombination, we show that the ionization depth in the planet's atmosphere reaches $\sim \frac{1}{2}$ mbar at the day/night terminator. Ionization leads to a slight weakening of the sodium feature. We present our baseline model, including ionization and clouds, which falls near the observational error bars. The sensitivity of our conclusions to the derived atmospheric temperature-pressure profile is discussed.
\end{abstract}

Subject headings: planetary systems — radiative transfer — stars: individual (HD 209458)

\section{INTRODUCTION}

The planet in the system HD 209458 has been an object of intense study since the first observations of its transit (Henry et al. 2000; Charbonneau et al. 2000). These observations led to a determination of the planet's radius and physical mass, confirming that it must be a gas giant. At wavelengths where the atmospheric opacity is high, the planet blocks more light, yielding a deeper transit light curve that makes the planet appear physically larger. The location and strength of these absorption features serve as diagnostics of the temperature and chemistry of the planet's atmosphere. Seager \& Sasselov (2000) predicted variations in the transmission spectrum of HD 209458b due to neutral $\mathrm{Na}$ and K and to singly ionized He. Brown (2001) explored effects such as ionization and winds and predicted large variations due to $\mathrm{H}_{2} \mathrm{O}, \mathrm{CO}$, and $\mathrm{CH}_{4}$ in the infrared. Hubbard et al. (2001) explored a variety of physical effects that could be found in transits, including refraction and the angular redistribution of photons due to Rayleigh scattering. These were found to be minimal for HD 209458b; Hubbard et al. (2001) went on to derive the planet radius as a function of wavelength from 300 to $2500 \mathrm{~nm}$.

Confirmation that absorption features due to the opacity of gaseous species could be observed with current technology was obtained by Brown et al. (2001). They observed the transit of HD 209458b from 581 to $638 \mathrm{~nm}$ with the STIS instrument on $H S T$ and obtained photometric accuracy near $100 \mu$ mag. This spectral region encompasses the $\mathrm{Na} D$ doublet at $589 \mathrm{~nm}$. Using these data, Charbonneau et al.

${ }^{1}$ Lunar and Planetary Laboratory, University of Arizona, Tucson, AZ 85721-0092; jfortney@lpl.arizona.edu,curtis@lpl.arizona.edu, hubbard@1pl.arizona.edu,jlunine@lpl.arizona.edu.

${ }^{2}$ Department of Astronomy and Steward Observatory, University of Arizona, Tucson, AZ 85721; sudarsky@as.arizona.edu,

burrows@as.arizona.edu.

${ }^{3}$ NASA Goddard Space Flight Center, Greenbelt, MD 20771; hubeny@tlusty.gsfc.nasa.gov.

${ }_{4}^{4}$ National Optical Astronomy Observatory, Tucson, AZ 85725.
(2002) found that the transit was deeper by $(2.32 \pm 0.57)$ $\times 10^{-4}$ in a narrow band centered on the star's $\mathrm{Na} \mathrm{D}$ lines than in adjoining bands at shorter and longer wavelengths. Importantly, the observed difference in transit depth in and out of the $\mathrm{Na} \mathrm{D}$ line is smaller than previously predicted (Charbonneau et al. 2002). The nominal model of Hubbard et al. (2001) predicted a difference between these two bands of $4.7 \times 10^{-4}$.

Charbonneau et al. (2002), using the models of Brown (2001), performed a parameter study to investigate a variety of possible reasons for this difference. These included a bulk underabundance of $\mathrm{Na}$, the sequestering of atomic $\mathrm{Na}$ in condensates and/or molecules, clouds very high in the planet's atmosphere, and photoionization of atomic $\mathrm{Na}$ due to the UV flux from the parent star. Barman et al. (2002) suggested that $\mathrm{Na}$ is not in local thermodynamic equilibrium (LTE). They speculated that this leads to a core reversal of the $589 \mathrm{~nm}$ absorption doublet in the planet's emission spectrum, but they did not calculate its effect on a transit light curve.

Here we explore the effects of cloud formation and ionization of Na in HD 209458b's atmosphere. We find that cloud opacity is the dominant effect that determines the depth of the $\mathrm{Na} \mathrm{D}$ feature, but ionization of $\mathrm{Na}$ also leads to a nonnegligible weakening of the $\mathrm{Na} \mathrm{D}$ doublet.

In our analysis, following Charbonneau et al. (2002), we define a narrow "in-feature" wavelength band as the wavelength range 588.7-589.9 nm, and an "out-of-feature" band as the combination of the 518.8-588.7 and 589.9$596.8 \mathrm{~nm}$ wavelength ranges. The in-feature band includes the wavelength extent of the easy-to-observe HD 209458A (stellar) $\mathrm{Na}$ absorption doublet. When quoting a depth for the Na D feature, we simply mean the difference in transit depth (the fractional change in flux) between the in-feature and out-of-feature bands at midtransit. The fractional change in flux at midtransit across the entire $581-638 \mathrm{~nm}$ wavelength range is -0.0164 , meaning $1.64 \%$ of the star's light is blocked. 


\section{TEMPERATURE-PRESSURE PROFILE}

The temperature-pressure $(T-P)$ profile for a close-in extrasolar giant planet (EGP) is flatter (more isothermal) than that of an isolated object with the same effective temperature because of heating of the outer atmosphere by the parent star. In the present work, we have employed TLUSTY (Hubeny \& Lanz 1995), a self-consistent atmosphere code that uses the discontinuous finite element (DFE) method for radiative transfer (Hubeny \& Lanz 1995; Castor, Dykema, \& Klein 1992). The atmosphere model is plane-parallel. Radiative equilibrium is achieved by adjusting the atmospheric temperature-density structure iteratively via the accelerated lambda iteration (ALI) method. The important physical quantities, including net flux, temperature, and density in every atmospheric depth zone, are converged to better than $0.1 \%$, and total energy is conserved to this same precision. For the deep convective layers a mixing-length prescription is used with a mixing-length parameter of 1 scale height.

For a close-in planet such as HD 209458b, an inner boundary flux corresponding to that of an isolated object with an effective temperature of $\sim 500 \mathrm{~K}$ or less results in an outer atmospheric structure that is independent of this inner boundary. The outer structure is determined by the stellar flux, the surface gravity, the atmospheric compositions, and the opacities (see Sudarsky, Burrows, \& Hubeny 2003; Burrows et al. 2001). The metallicity of the parent star has been shown to be consistent with solar abundances (Mazeh et al. 2000), and we use these abundances when modeling the planet's atmosphere.

The $T$ - $P$ profile of HD 209458b's atmosphere that we derive is shown in Figure 1. We use a surface gravity of 980 $\mathrm{cm} \mathrm{s}^{-2}$ for the planet. We find that HD 209458b is a "Class V" planet as defined in Sudarsky, Burrows, \& Pinto (2000) and Sudarsky et al. (2003), meaning that silicate and iron clouds occur at altitudes in the planet's atmosphere relevant to spectroscopic observations. We model the location, distribution, and size of cloud particles using the model of

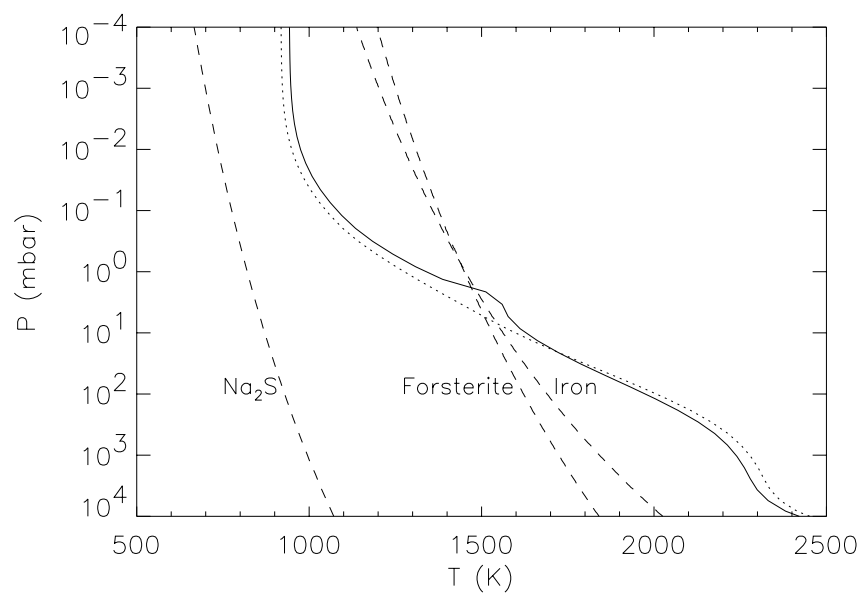

FIG. 1. - T-P profile for the atmosphere of HD $209458 \mathrm{~b}$ (solid line). The dotted line shows the $T-P$ profile we derive without the opacity due to clouds. The condensation curves for $\mathrm{Na}_{2} \mathrm{~S}$, forsterite $\left(\mathrm{Mg}_{2} \mathrm{SiO}_{4}\right)$, and iron are shown as dashed lines. When cloud opacity is taken into account, the forsterite cloud base moves from 5.4 to $1.8 \mathrm{mbar}$. There is a wide range of possible $T-P$ profiles for which forsterite and iron clouds form high in the planet's atmosphere but $\mathrm{Na}$ remains in atomic form. We used a surface gravity of $980 \mathrm{~cm} \mathrm{~s}^{-2}$ for the planet.
Cooper et al. (2003). As seen in Figure 1, the cloud bases of forsterite $\left(\mathrm{Mg}_{2} \mathrm{SiO}_{4}\right)$ and iron $(\mathrm{Fe})$ are at the several-millibar level. The effect of these clouds on the transit is discussed in $\S 4$.

In calculating the $T-P$ profile, we assume that incoming stellar energy is absorbed and redistributed evenly on the day side, but is not redistributed onto the night side. In a recent paper, Showman \& Guillot (2002) find that redistribution of energy around the entire planet may be inefficient, leading to $\mathrm{a} \sim 500 \mathrm{~K}$ effective temperature difference between the planet's day and night sides. The transit observation is sensitive to the planet's atmosphere on the limb, which could have a $T-P$ profile slightly cooler than we predict here. (Note that the atmospheric circulation code of Showman \& Guillot 2002 is not able to calculate $T$-P profiles for the planet.) Based on this possibly large temperature difference, Guillot \& Showman (2002) make the point that if HD 209458 b does have a $500 \mathrm{~K}$ effective temperature contrast between the night and day sides, condensates could form on the night side and tie up $\mathrm{Na}$, thereby weakening the $\mathrm{Na} D$ absorption feature. Note, however, that the "cold" $T$ - $P$ profile used in Hubbard et al. (2001), with a temperature $400 \mathrm{~K}$ cooler at 1 mbar than seen in Figure 1, still has a $589 \mathrm{~nm} \mathrm{Na}$ feature depth of $3.2 \times 10^{-4}$, deeper than observed.

Our $T-P$ profile differs significantly from that calculated in the recent paper on HD 209458b by Barman et al. (2002). We find a temperature of $1280 \mathrm{~K}$ at $1 \mathrm{mbar}$ and $950 \mathrm{~K}$ at $10 \mu$ bar. Examination of Figure 1 in Barman et al. (2002) shows temperatures of $\sim 1790 \mathrm{~K}$ at 1 mbar and $1630 \mathrm{~K}$ at $10 \mu$ bar. The reasons for these large differences are unclear, but they could be due to differing opacities, stellar flux, or planet surface gravity. Our $T-P$ profile is closer to those for HD 209458b discussed by Seager \& Sasselov (2000) and Richardson et al. (2003). These differences are significant, and we are led to very different conclusions concerning the physical processes of importance for the $\mathrm{Na} \mathrm{D}$ transit depth. As with late L and T dwarfs, our HD 209459b atmosphere is free of metals such as $\mathrm{Mg}, \mathrm{Al}, \mathrm{Ca}, \mathrm{Fe}$, and $\mathrm{Si}$, which have been incorporated into condensates. Barman et al. (2002) find an atmosphere that is not cool enough to allow for the formation of these condensates, which leaves their atmosphere rich in heavy metals in atomic form. Because of the high UV opacity of free metals that remain in the atmosphere and absorb stellar radiation, Barman et al. (2002) conclude that ionization of $\mathrm{Na}$ at pressures greater than 1 $\mu$ bar is negligible. Our model temperatures allow deeper ionization of $\mathrm{Na}$ in the atmosphere of the planet.

\section{TRANSIT CALCULATIONS}

The methods we use for calculating the effects of absorption by gaseous species, absorption and scattering by condensates, Rayleigh scattering, and refraction are essentially identical to those described in Hubbard et al. (2001), although we remove the simplification that the opacityversus-pressure profile is the same around the entire planet.

We calculate the slant optical depth through the planet's atmosphere, $\tau$, from the expression

$$
\tau=\int_{-\infty}^{r} \frac{r^{\prime \prime} d r^{\prime \prime} \sigma\left(r^{\prime \prime}\right) N\left(r^{\prime \prime}\right)}{\left(r^{\prime \prime 2}-r^{2}\right)^{1 / 2}}+\int_{r}^{\infty} \frac{r^{\prime \prime} d r^{\prime \prime} \sigma\left(r^{\prime \prime}\right) N\left(r^{\prime \prime}\right)}{\left(r^{\prime \prime 2}-r^{2}\right)^{1 / 2}}
$$

where $r$ is the impact parameter of a light ray, $r^{\prime \prime}$ is the 


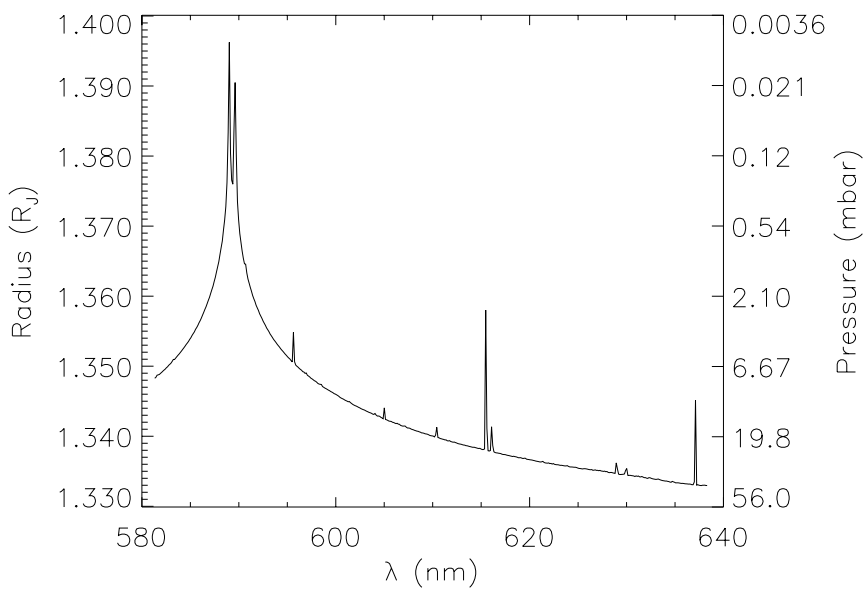

FIG. 2.-Planetary radius vs. wavelength, also showing the pressure at the planet's limb corresponding to the derived radius, for a neutral cloudless atmosphere. ( $1 R_{\mathrm{J}}=71,492 \mathrm{~km}$, Jupiter's equatorial radius at 1 bar.) The radius values are the radii derived from calculating the apparent crosssectional area of the spherical planet, and this radius is essentially identical to the radius at a slant optical depth of $\frac{2}{3}$. The wavelengths shown encompass the Brown et al. (2001) observations. During the transit, an observer is sensitive to pressures from tens of millibars to $\sim 10 \mu \mathrm{bar}$. The average pressure across this wavelength band is $15 \mathrm{mbar}$.

magnitude of the three-dimensional vector position of an atmospheric point from the planet's center, $\sigma$ is the absorption cross section per molecule, and $N$ is the number density of molecules in the atmosphere. Two terms in the $\tau$ expression are needed because $\sigma\left(r^{\prime \prime}\right)$ is different on the day and night sides.

As before, we use the planetary and stellar properties from Brown et al. (2001). Figure 2 shows, for a cloudless atmosphere with no ionization, the radius as a function of wavelength over the range covered by the Brown et al. (2001) and Charbonneau et al. (2002) observations. The auxiliary axis of Figure 2 shows the pressure at the terminator to which this radius corresponds; the figure thus indicates the pressure range probed by the transit of HD 209458b.

\section{CLOUD MODEL}

\subsection{Location, Distribution, and Opacity}

The $T$ - $P$ profile we derive is warmer than the ad hoc profiles studied in Hubbard et al. (2001). Because of this, we find forsterite and iron clouds higher in the planet's atmosphere. If we examine the forsterite cloud in Figure 1, for the $T-P$ profile without cloud opacity, the cloud base is at 5.4 mbar. The opacity due to clouds slightly warms the planet's atmosphere, especially at the location of the clouds, moving the cloud base farther out, to 1.8 mbar. Predicting the exact location of the cloud base is a nontrivial problem, and in a fully self-consistent calculation of cloud location, one would need to iterate many times successively between the cloud code and atmosphere code. Since cloud opacity is a strong function of particle size, and the predicted particle sizes are altered because of a change in the cloud base and as function of pressure within the cloud itself (as described below), this step is challenging. Since uncertainties remain in the modeling of clouds and the effects of redistribution of energy on the $T-P$, we do not attempt this fully self- consistent treatment. Instead, our T-P profile shown in Figure 1 incorporates the opacity of the cloud with the location of the base given by the point of intersection with the condensation curve and with a cloud particle size given by the modal size for the entire cloud. The particle number density falls off exponentially for 1 scale height.

The cloud model of Cooper et al. (2003) predicts particle radii in this radiative region for the iron cloud that range from tens of microns at the base to less than $1 \mu \mathrm{m}$ at the cloud tops. In the forsterite cloud, all particles are 0.05-0.5 $\mu \mathrm{m}$ in size. The cross sections are calculated as in Sudarsky et al. (2003). For these submicron particles, scattering dominates over absorption, and the opacity of the forsterite cloud is significantly larger than that of the iron cloud. The Cooper et al. (2003) model predicts a distribution of cloud particle sizes and densities as a function of pressure in the atmosphere. Therefore, we calculate the cross sections for the cloud as a function of pressure at many pressures within the cloud and put no artificial cap on the cloud top. While it is well known that understanding the vertical distribution of cloud material is important, it is of even greater importance for the transit spectroscopy of EGP atmospheres, because cloud opacity can be so great for our slant viewing geometry.

For HD 209458b, we find a forsterite cloud with significant vertical extent, with a large slant optical depth ( $\tau \sim 500$ at the base) that does not fall to $\tau \sim \frac{2}{3}$ until 1.9 gas scale heights above the cloud base. Even though $99 \%$ of the cloud's mass is found within 1 gas scale height, a nonnegligible population of small, highly scattering cloud particles continues above this level, giving rise to a forsterite cloud with a significant slant optical depth up to $\sim 1.9$ scale heights. Since our predicted forsterite cloud is in a region of interest for HD 209458b's transit spectroscopy, the vertical extent of the cloud is of great importance. It seems likely that any clouds seen during transit spectroscopy will be in a radiative (quiescent) region, in which case the cloud particles will likely remain small, in contrast to the large sizes (10-200 $\mu \mathrm{m})$ predicted for convective regions. Given current uncertainties in cloud modeling (see Cooper et al. 2003), much work still needs to be done in this area. While Seager \& Sasselov (2000) were the first to note the importance of clouds in determining the depth of transit absorption features, here we note that understanding the vertical distribution of clouds is yet a further challenge in EGP transit spectroscopy.

In this narrow spectral region $(581-638 \mathrm{~nm})$, the cross sections have virtually no wavelength dependence. Thus, clouds lead to a wavelength-independent absorption of stellar light greater than that of the gaseous species alone, leading to a uniform increase in the planetary radius across much of the wavelength band. In the core of the $\mathrm{Na} \mathrm{D}$ feature, where the slant optical depth reaches unity at much lower pressures, the clouds have no effect. This leads to a decrease of the contrast in transit depth between the in-feature and out-of-feature bands. Figure 3 is analogous to Figure 2, but in this case it shows the planet's radius and pressure at the terminator as a function of wavelength, for our atmosphere including clouds, with the base at 5.4 mbar. In both cases, the planet's radius at 1 bar has been adjusted so that the transit depth remains $1.64 \%$.

Figure 4 shows the transit depth versus wavelength for a neutral cloud-free atmosphere and a neutral atmosphere 


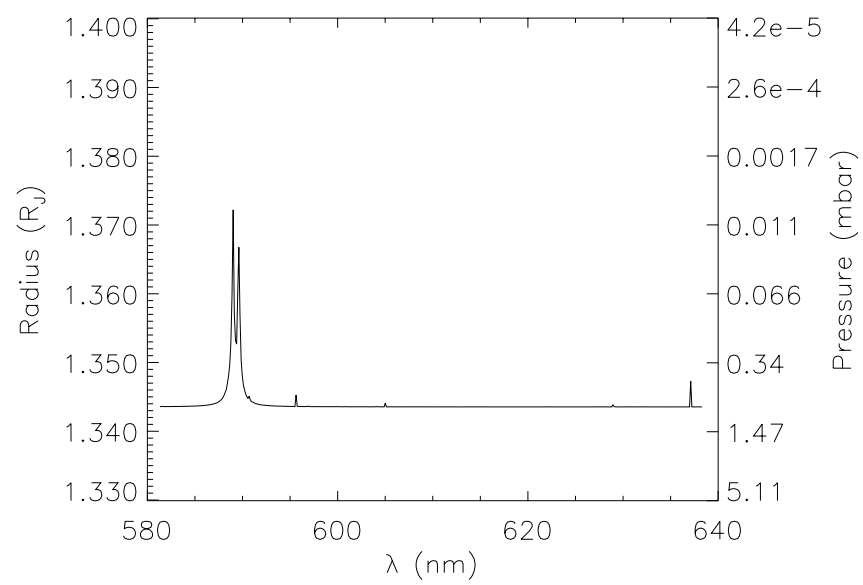

FIG. 3.-Similar to Fig. 2, but for a neutral atmosphere with a forsterite cloud base of 5.4 mbar. The pressure axis changes because of the different opacity in the planet's atmosphere, as compared to that in Fig. 2. The clouds lead to a wavelength-independent increase in planetary radius. The average pressure across this wavelength band is $1 \mathrm{mbar}$.

with the predicted clouds. The cloud-free model gives a feature depth of $5.20 \times 10^{-4}$, deeper than that obtained by Hubbard et al. (2001) because of our warmer $T-P$ profile. The model with clouds decreases this depth significantly. With the forsterite cloud base at 5.4 mbar, the depth is $2.42 \times 10^{-4}$, while if the cloud base is at 1.8 mbar the depth is $0.93 \times 10^{-4}$. The depth is actually smaller than the Charbonneau et al. (2002) observations, $(2.32 \pm 0.57) \times 10^{-4}$, indicating that if clouds form too high in the planet's atmosphere they can dominate over the opacity of gaseous species.

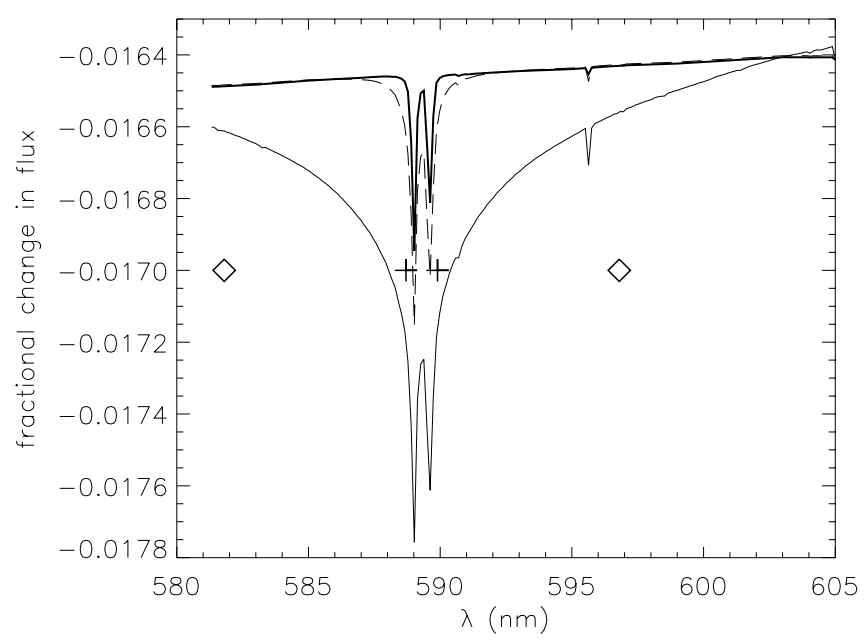

FIG. 4.-Transit depth (at midtransit) vs. wavelength for several models. Curves appear offset because the transit depth is required to be a fractional change in flux of -0.0164 from 581 to $638 \mathrm{~nm}$, the full Brown et al. (2001) observation band. The two plus signs mark the wavelength extent of the infeature band from Charbonneau et al. (2002), while the wavelengths between the plusses and diamonds, on either side of the in-feature band, make up the out-of-feature band. The observed difference in transit depth between these bands is $(2.32 \pm 0.57) \times 10^{-4}$. Shown are neutral models with and without clouds. The $\mathrm{Na}$ feature depth for the neutral cloudless model (thin solid line) is $5.20 \times 10^{-4}$. The feature depth is $2.42 \times 10^{-4}$ for a forsterite cloud base at 5.4 mbar (dashed line) and $0.93 \times 10^{-4}$ for a cloud base at 1.8 mbar (thick solid line).

\subsection{Sensitivity of Results}

Figure 1, which shows the $T-P$ profile, along with the pressure axes on Figures 2 and 3, highlights how sensitively the $\mathrm{Na} \mathrm{D}$ feature depth depends on the location of clouds. Moving the cloud base $\sim 1$ gas scale height can cause an appreciable difference in the $\mathrm{Na} \mathrm{D}$ feature strength. As Figure 1 and the transit depths for the two models with clouds show, a change in the pressure of the cloud base of this amount would happen with fairly minor deviations from our derived $T-P$ profile for the planet's atmosphere. If the cloud base occurs at $1.8 \mathrm{mbar}$, rather than $5.4 \mathrm{mbar}$, the feature depth moves from comfortably within the error bars to significantly below. Even higher clouds (warmer $T-P$ profiles) would lead to even weaker $\mathrm{Na} \mathrm{D}$ features. At several hundred kelvins warmer, no condensate clouds would form, as is the case in the model of Barman et al. (2002). Perhaps the most important issue is that changing our assumption of how absorbed stellar radiation is redistributed around the planet causes nonnegligible changes to the planet's $T$ - $P$ profile (see, e.g., Sudarsky et al. 2003).

We turn next to the role of ionization and treat the case in which the opacity profile varies over the planet. We find that the depth to which the $\mathrm{Na}$ is ionized changes with angle from the planet's subsolar point. In Hubbard et al. (2001), $\mathrm{Na}$ ionization by UV irradiation was ignored. Here we find that ionization of $\mathrm{Na}$ probably has a secondary but nonnegligible effect on the observed $\mathrm{Na} \mathrm{D}$ feature.

\section{A SIMPLE NON-LTE SODIUM IONIZATION MODEL}

The proximity to the parent star subjects the transiting planet to large amounts of ionizing radiation, making equilibrium chemistry treatments inadequate. In particular, $\mathrm{Na}$ ionization alters the profile of neutral $\mathrm{Na}$. This is crucial, since the $\mathrm{Na} \mathrm{D}$ line and its transit signature are features of the neutral state. A rigorous study of the ionization of sodium in the atmosphere of HD 209458b would require sophisticated non-LTE and nonequilibrium photochemical modeling. However, most of the necessary atomic and molecular data (in particular, collisional rates and dissociation parameters) are either poorly known or completely unknown. Therefore, we attempt to calculate the effects of ionization using simple, parametric models.

Barman et al. (2002) have performed a detailed study of non-LTE effects on excitation and ionization of $\mathrm{Na} \mathrm{I}$ in the atmosphere of HD 209458b. However, they find that the $\mathrm{UV}$ opacity is dominated by atomic metals $(\mathrm{Mg}, \mathrm{Al}, \mathrm{Ca}, \mathrm{Fe}$, and $\mathrm{Si}$ ). Because of the much cooler temperatures we find, these elements are tied up in condensates and are thus depleted from the outer layers of the atmosphere of HD 209458b. Therefore, they cannot shield sodium from intense stellar ionizing radiation. Moreover, Barman et al. (2002) had to parameterize the corresponding collisional rates, and they found that free electrons are only due to ionization of potassium.

In view of all uncertainties in the atomic and molecular parameters, especially the UV opacities, we feel that it is not warranted to perform a detailed non-LTE study. Instead, we devise a simple, non-LTE analytical model that allows us to study the effects of various uncertainties in relevant quantities (opacities, free electron densities, collisional rates) on the degree of ionization of sodium in a straightforward way. 
We find that much of the outer layers of the atmosphere $\left(P \lesssim \frac{1}{2} \mathrm{mbar}\right.$ ), where $\mathrm{Na}$ I has the greatest likelihood of having non-LTE level populations, is ionized by the stellar UV flux. We also calculate the change in the ionization depth as a function of angle from the substellar point, which to this point has not been investigated in the literature.

\subsection{Description of the Model}

We assume that both neutral and ionized sodium are represented by one-level atoms, with populations (number densities) $n_{1}$ and $n_{2}$. The third unknown of the problem is the electron density, $n_{e}$. The three equations that specify these unknown number densities are the particle conservation equation,

$$
n_{1}+n_{2}=N_{\mathrm{Na}},
$$

charge conservation equation,

$$
n_{e}=n_{2}+\alpha N_{\mathrm{Na}}+\beta n_{2},
$$

and the statistical equilibrium equation for sodium,

$$
\begin{aligned}
n_{1}\left(R_{12}+n_{e} C_{12}+n_{\mathrm{H}_{2}}\right. & \left.C_{12} e_{\mathrm{H}_{2}}\right) \\
& =n_{2}\left(R_{21}+n_{e} C_{21}+n_{\mathrm{H}_{2}} C_{21} e_{\mathrm{H}_{2}}\right),
\end{aligned}
$$

where $N_{\mathrm{Na}}$ is the total sodium number density. In equation (3), we assume that the total number of electrons is given by the total number of ionized sodium atoms, plus a contribution from an unspecified source that is given through some fraction $\alpha$ of the total number of sodium atoms (that is, it scales as the total density), plus another empirical contribution that scales as ionized sodium, with a multiplicative factor $\beta$. Setting $\alpha=\beta=0$ stipulates that free electrons are provided only by ionization of sodium.

In the statistical equilibrium equation (eq. [4]), $R_{12}$ and $R_{21}$ are the photoionization and radiative recombination rates, respectively; $n_{e} C_{12}$ and $n_{e} C_{21}$ are the collisional ionization and recombination (three-body recombination) rates with free electrons. Since the dominant species in the atmosphere is $\mathrm{H}_{2}$, we have to consider ionization of sodium by collisions with $\mathrm{H}_{2}$, and its inverse process as well. In the absence of any knowledge of relevant rates, we assume, similarly to Barman et al. (2002), that the collision rate is given by a scaled electron rate with some factor $e_{\mathrm{H}_{2}}$. It is reasonable to consider this factor to be the ratio of reduced masses of the $\mathrm{Na}+\mathrm{H}_{2}$ pair to that of $\mathrm{Na}+$ electron, which is about $1 / 60$. However, we keep $e_{\mathrm{H}_{2}}$ as a free parameter, allowing us to study the effects of the $\mathrm{H}_{2}$ collision efficiency on $\mathrm{Na}$ ionization.

By detailed balancing arguments (see, e.g., Mihalas 1978), the inverse rates are given by

$$
C_{21}=C_{12}\left(n_{1} / n_{2}\right)^{*}=n_{e} \phi(T) C_{12},
$$

where $\left(n_{1} / n_{2}\right)^{*}$ denotes the LTE value and $\phi$ is the SahaBoltzmann factor, given by

$$
\phi(T)=2.0706 \times 10^{-16}\left(g_{1} / g_{2}\right) T^{-3 / 2} \exp \left(h \nu_{0} / k T\right),
$$

where $T$ is the temperature, $g_{1}$ and $g_{2}$ the statistical weights ( $g_{1}=2, g_{2}=1$ for sodium), $\nu_{0}$ the ionization frequency, and $h$ and $k$ the Planck and Boltzmann constants.
The radiative rates are given by

$$
R_{12}=\frac{4 \pi}{h} \int_{\nu_{0}}^{\infty} \frac{\sigma(\nu)}{\nu} J_{\nu} d \nu
$$

and

$$
R_{21}=n_{e} \phi(T) \frac{4 \pi}{h} \int_{\nu_{0}}^{\infty} \frac{\sigma(\nu)}{\nu}\left(\frac{2 h \nu^{3}}{c^{2}}+J_{\nu}\right) \exp (-h \nu / k T) d \nu,
$$

where $\sigma(\nu)$ is the photoionization cross section and $J_{\nu}$ is the mean intensity of radiation. However, equation (8) takes into account only recombinations to the ground state of $\mathrm{Na}$ I. In reality, recombinations can occur to any state, and at low temperatures these processes are very efficient. We therefore take the recombination rate to be

$$
R_{21}=\frac{5.461 \times 10^{-12}}{\sqrt{T / T_{0}}\left(1+\sqrt{T / T_{0}}\right)^{0.825}},
$$

with $T_{0}=307.7 \mathrm{~K}$, as given by Verner \& Ferland (1996).

The radiation intensity should in principle be determined by solving the radiative transfer equation. We could do so, but in view of gross uncertainties in relevant UV opacities, this is not warranted. Instead, we simply assume that the specific intensity is given as a sum of attenuated stellar irradiation intensity plus a thermal component given by the Planck function at the local temperature. (This part is negligible at surface layers of interest, but gives the correct behavior of intensity at depth.) To allow for uncertainties in UV opacities, we introduce another multiplicative factor, $w_{\text {add, }}$ which represents an additional attenuation of radiation, perhaps by an unspecified opacity source.

The intensity as a function of depth is given by

$$
I_{\nu \mu}\left(\tau_{\nu}\right)=w_{\text {add }} I_{\nu \mu}^{\text {inc }} \exp \left(-\tau_{\nu} \mu\right)+B_{\nu}(T),
$$

where $\mu$ is the cosine of the angle with respect to the surface normal, $\tau_{\nu}(P)$ is the optical depth (in the normal direction), and $I_{\nu \mu}^{\mathrm{inc}}$ is the specific intensity of the stellar radiation at the top of the planetary atmosphere. We use a Kurucz model for a G0 V star with a dilution factor of $w=1.4 \times 10^{-2}$ because of the planet-star distance. For simplicity, we assume isotropic irradiation, which gives

$$
J_{\nu} \equiv \frac{1}{2} \int_{-1}^{1} I_{\nu \mu} d \mu=\frac{1}{2} w_{\mathrm{add}} I_{\nu}^{\mathrm{inc}} E_{2}(\tau)+B_{\nu}(T),
$$

where $E_{2}$ is the second exponential integral.

The electron collisional ionization rate is assumed to be given by Seaton's formula,

$$
C_{12}=1.55 \times 10^{13} T^{-1 / 2} \bar{g} \sigma\left(\nu_{0}\right) \exp \left(-u_{0}\right) / u_{0},
$$

where $\sigma\left(\nu_{0}\right)$ is the photoionization cross section at the edge, $\bar{g}=0.1$, and $u_{0}=h \nu_{0} / k T$.

\subsection{UV Opacities}

Since we find no free heavy elements in atomic form above the clouds in which these elements are sequestered, any opacity that may affect $\mathrm{Na}$ ionization must be provided by molecular species. We have searched the literature and found only two equilibrium species that contribute significantly to the opacity in the near UV- $\mathrm{H}_{2} \mathrm{O}$ (Yoshino et al. 


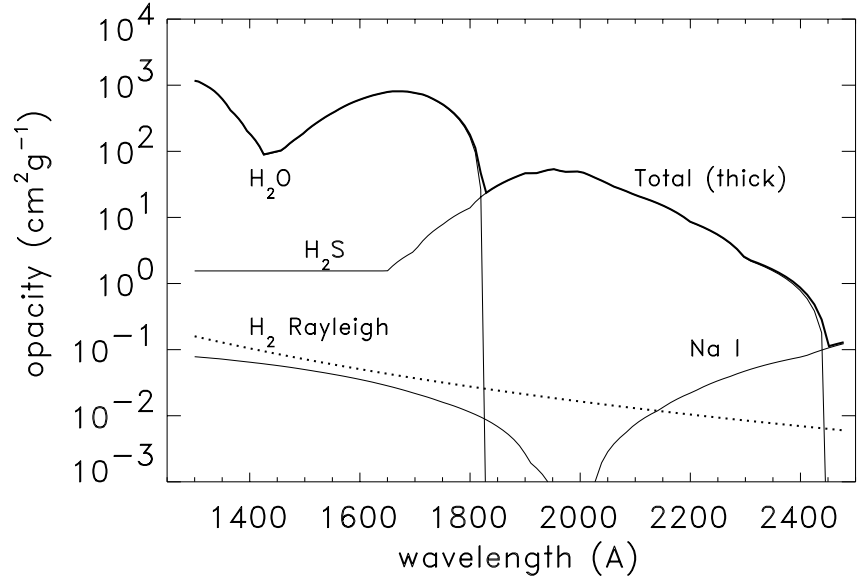

FIG. 5.-Opacity at the outer layers of the atmosphere of HD 209458b.

1996) and $\mathrm{H}_{2} \mathrm{~S}$ (Lee, Wang, \& Suto 1987). Rayleigh scattering on $\mathrm{H}_{2}$ is much less important at this range. For both $\mathrm{H}_{2} \mathrm{O}$ and $\mathrm{H}_{2} \mathrm{~S}$, data are only available at $295 \mathrm{~K}$. We plot in Figure 5 the total opacity, plus the individual contributions of $\mathrm{H}_{2} \mathrm{O}, \mathrm{H}_{2} \mathrm{~S}, \mathrm{H}_{2}$, and a maximum possible contribution of $\mathrm{Na}$ I (that is, assuming that sodium is completely neutral) at upper layers of the atmosphere of HD 209458b, as they follow from the atmosphere model. The corresponding $\mathrm{Na}$ I photoionization cross section is taken from the Opacity Project database (Cunto et al. 1993).

\subsection{Representative Results}

The basic set of equations (eqs. [2]-[4]), together with auxiliary expressions (eqs. [5]-[11]), can be cast into a single cubic equation for $n_{2} / N_{\mathrm{Na}}$. All the necessary quantities, $T$, $N_{\mathrm{Na}}, n\left(\mathrm{H}_{2}\right), n\left(\mathrm{H}_{2} \mathrm{O}\right)$, and $n\left(\mathrm{H}_{2} \mathrm{~S}\right)$, as functions of depth, are provided by our model atmosphere for HD 209458b.

Our nominal model, without any empirical modifications, uses $\alpha=\beta=0, E_{\mathrm{H}_{2}}=1 / 60$, and $w_{\text {add }}=1$. The depth dependence of $\mathrm{Na}$ ionization for this model is shown in Figure 6. This calculation corresponds to the planet's sub-

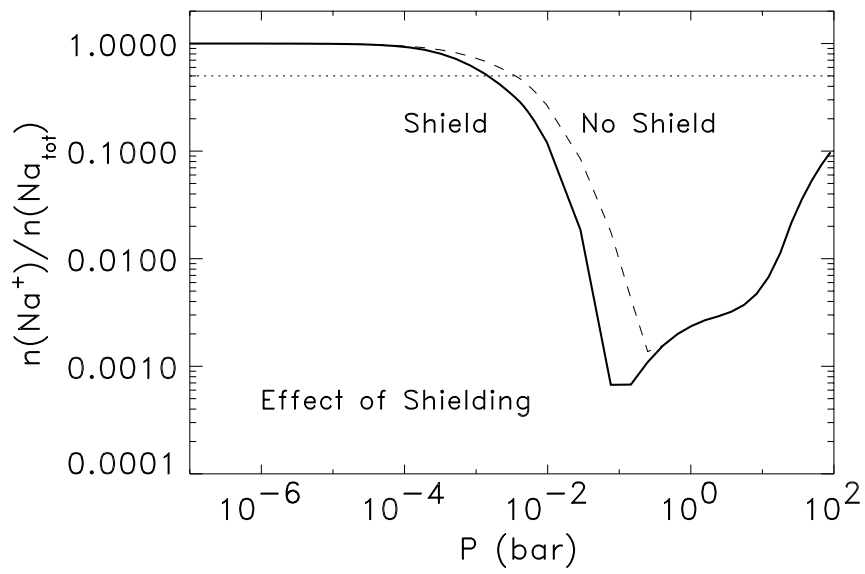

FIG. 6.-Influence of shielding on the Na ionization depth due to UV absorption by $\mathrm{H}_{2} \mathrm{O}, \mathrm{H}_{2} \mathrm{~S}$, and Rayleigh scattering. The thick solid line is for our nominal model, while the dashed line is for the nominal model, but with $\mathrm{Na}$ able to absorb all ionizing radiation. The horizontal dotted line shows the location of $50 \% \mathrm{Na}$ ionization.

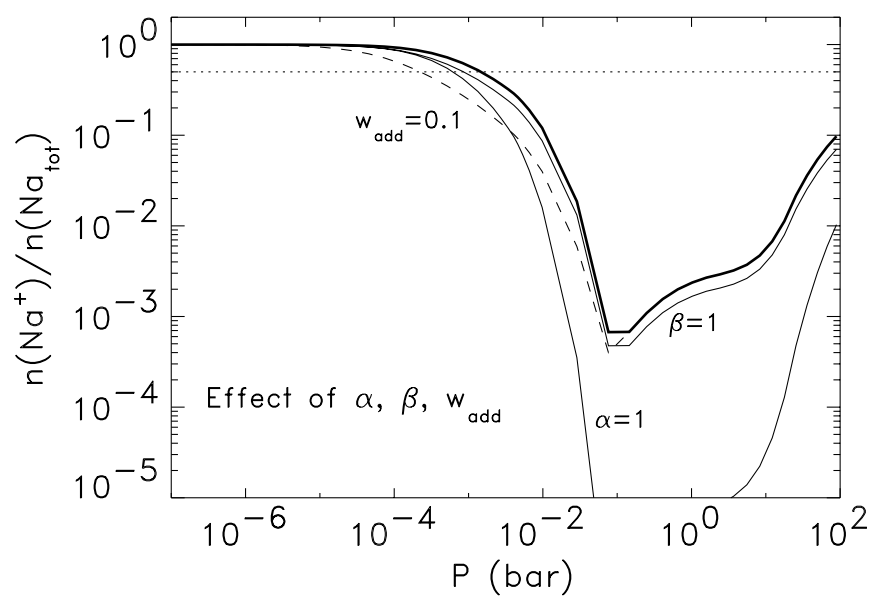

FIG. 7.- Influence on the ionization depth of factors $\alpha, \beta, w_{\text {add }}$, leaving other parameters at their default values. As described in the text, these factors are meant to show the sensitivity of our results to possible unknown additional free electrons or additional UV opacity. The thick solid curve shows our default calculated ionization depth curve $(\alpha=\beta=$ $\left.e_{\mathrm{H}_{2}}=0, w_{\text {add }}=1\right)$. The thin solid curves are for $\alpha=1$ and $\beta=1$, while the dashed curve is for $w_{\text {add }}=0.1$. The horizontal dotted line shows the location of $50 \% \mathrm{Na}$ ionization. All these factors would push the ionization depth out to lower pressures.

solar point. For the nominal model, the pressure at which the number densities of $\mathrm{Na}$ I and $\mathrm{Na}^{+}$are equal is $1.6 \mathrm{mbar}$. At greater pressures, neutral $\mathrm{Na}$ dominates. Deep in the planet's atmosphere, at pressures greater than 100 bar, ionization of $\mathrm{Na}$ again becomes appreciable because of the higher temperatures. Also shown is the ionization depth dependence without shielding due to the other UV absorbers. The pressure at which $\mathrm{Na}$ is $50 \%$ ionized increases to 3 mbar.

We next test our predictions for $\mathrm{Na}$ ionization depth by varying several of the parameters mentioned above. These trials can be seen in Figure 7. In the first case $\alpha=1$, which represents the case in which there is another donor, with a total abundance equal to that of sodium, which is fully ionized. The model with $\beta=1$ represents an additional empirical donor of free electrons that has the same abundance as ionized sodium. The model with $w_{\text {add }}=0.1$ represents the situation in which unknown UV absorbers leave only $10 \%$ of the calculated incoming ionizing flux available to ionize $\mathrm{Na}$. The electron donor factors seem like reasonable upper limits because species more abundant than $\mathrm{Na}$ in the planet's atmosphere tend to have 2-3 times larger ionization energies and consequently are difficult to ionize. These factors have a modest effect on the pressure at which $\mathrm{Na}$ is $50 \%$ ionized. Changing the value of $e_{\mathrm{H}_{2}}$ (which characterizes the $\mathrm{H}_{2}$ collision efficiency) by factors of even 100 had negligible effects on the ionization depth for pressures less than 100 mbar.

\subsection{Ionization on the Planet's Limb}

The important drawback of the ionization model outlined above is that the calculations are representative of the subsolar point only. We are interested in ionization at the planet's limb. At a given location in the planet's atmosphere at an angle $\theta$ from the subsolar point, the absorbed radiation of a given surface area will be diluted by a factor $\cos \theta$, meaning the ionization depth will not be as great. Even this 
geometric view is too simple, as it would imply no photoionization at the planet's terminator. To overcome this shortcoming, we have employed a somewhat different ionization model that can predict the ionization depth versus angle around the planet.

In this angle-dependent, two-dimensional ionization model (which we call model 2, while the prior model is model 1), we assume that incoming photons with energy greater than $5.14 \mathrm{eV}$ (the ionization energy of $\mathrm{Na}$ ) and less than $11.2 \mathrm{eV}$, the energy at which significant $\mathrm{H}_{2}$ absorption begins, ionize atomic $\mathrm{Na}$. $\mathrm{Na}^{+}$is the only ionized species, and consequently the number of free electrons is equal to the number of $\mathrm{Na}^{+}$ions. We use two- and three-body radiative recombination coefficients from Verner \& Ferland (1996) and Gudzenko et al. (1974). Incoming photons at some oblique angle relative to the surface can ionize all the $\mathrm{Na}$ along a chord until, at some pressure, most ionizing photons (per second) have been used up, and recombinations (per second) can match ionizations (per second). This is the ionization depth. Much as for a Strömgren sphere, we find a sharp boundary between regions of the planet's atmosphere with complete ionization and with no ionization. (Calculations from model 1 find a more gradual onset of neutral $\mathrm{Na}$ with depth.) We calculate the depth to which the atmosphere is ionized at several hundred angular positions from the planet's substellar point. We find that $\mathrm{Na}$ is ionized to a depth of $\sim 12$ mbar at the planet's substellar point (this was 1.6 mbar for model 1 with shielding due to UV absorbers and 3 mbar without), and as one moves away from this point, the pressure to which $\mathrm{Na}$ is ionized decreases. At the terminator $\left(90^{\circ}\right.$ from the subsolar point) the ionization depth is 1.7 mbar. Figure 8 shows representative calculations for the change in ionization depth with angle near the terminator. At an angle of $\sim 10^{\circ}$ past the terminator, no Na is ionized. The model possesses cylindrical symmetry about the planet's subsolar-antisolar axis. The removal of the opacity of atomic $\mathrm{Na}$ in the $10 \mathrm{mbar}-1 \mu \mathrm{bar}$ region of the atmosphere produces a negligible difference in the $T$ - $P$ profile.

The column density of atmosphere through which photons travel until one reaches the ionization depth of $\mathrm{Na}$ can

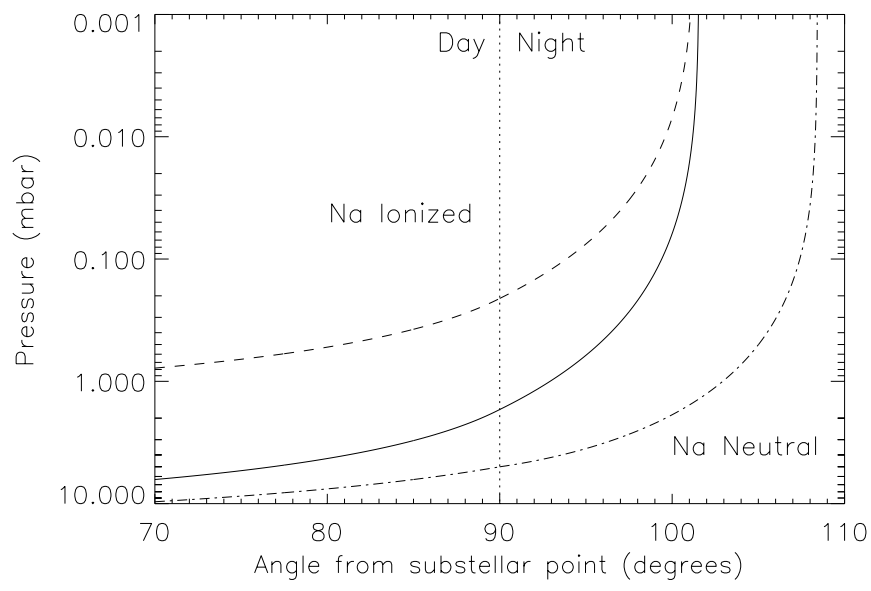

FIG. 8. - Sodium ionization depth vs. angle from the planet's substellar point. The vertical line at $90^{\circ}$ indicates the terminator. The dashed curve is for model 1, while the solid curve is for model 2 . All Na is ionized to the left of this curve and neutral to the right of the curve. The dot-dashed curve shows an increased ionization depth at every angle, an example of an ionization depth curve that would be necessary to match the observations in the absence of any cloud opacity.

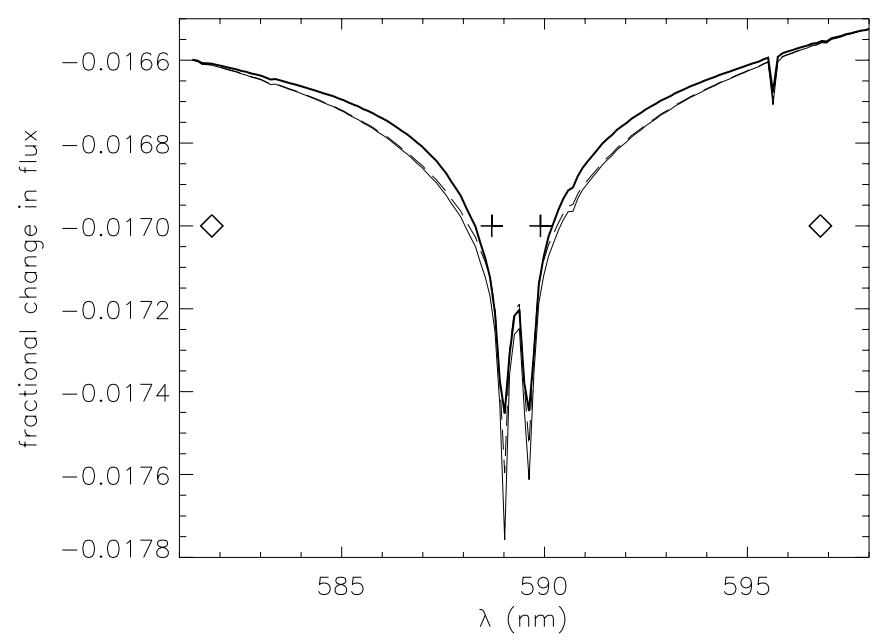

Fig. 9.-Transit depth (at midtransit) vs. wavelength for several models, similar to Fig. 4. The Na feature depth for a neutral cloudless model (thin solid line) is $5.20 \times 10^{-4}$. For the ionized cases, the feature depth is $4.72 \times 10^{-4}$ for model 1 (dashed line), and $4.83 \times 10^{-4}$ for model 2 (thick solid line). Even though the ionization depth is greater for model 2 (see Fig. 8 and the text), the in- and out-of-feature difference is greater, because in model 2 the Na wings are affected, but in model 1 they are not.

be calculated at the subsolar point for model 1 and at any angle, including the subsolar point, for model 2 . Using the ratio of the column depths of the two models at the subsolar point, one can scale the results from model 2 to calculate the ionization depth around the planet for model 1. Figure 8 shows the ionization depth versus angle for the scaled model 1 results.

Figure 9 depicts how ionization leads to a shallower transit depth. The differences between the Charbonneau et al. (2002) in-feature and out-of-feature bands are, for model $1,4.72 \times 10^{-4}$ and, for model $2,4.83 \times 10^{-4}$. Neither of these models include the predicted clouds, so that the relatively minor effect due to ionization can be shown clearly. Figure 8 shows a model in which we increased the pressure level to which $\mathrm{Na}$ is ionized, in order to gauge how much ionization is needed to match the Charbonneau et al. (2002) observations without the opacity of clouds. The feature depth for this model is $2.87 \times 10^{-4}$.

\section{CONCLUSIONS}

We find that HD 209458b's atmosphere is cool enough for most heavy elements to have been incorporated into condensates but still warm enough for $\mathrm{Na}$ to remain in atomic form. As seen in Figure 1, there is a range of $\sim 600 \mathrm{~K}$ in the mbar region of the planet's atmosphere where this situation holds. High clouds arise naturally in the planet's atmosphere near $\sim 1-5$ mbar. Although the abundances of condensable vapor of the chief cloud-forming species are small, the particles that form in this radiative region are small and have large scattering cross sections. Clouds lead to an increase in the apparent planet radius outside of the $\mathrm{Na} \mathrm{D}$ core, and on their own they could easily explain the observations. In addition, clouds can be so high that the predicted sodium feature is less than observed.

With our simple non-LTE model, we have shown that stellar UV flux will ionize $\mathrm{Na}$ to $\sim \frac{1}{2}$ mbar on the limb in the planet's atmosphere, reducing the strength of the $589 \mathrm{~nm}$ 
absorption feature. The change in the $\mathrm{Na} \mathrm{D}$ feature due to ionization is fairly insensitive to the derived $T-P$ profile. Ionization is a secondary effect, and it alone cannot explain the weak $\mathrm{Na} \mathrm{D}$ features. This conclusion was also reached by Charbonneau et al. (2002) in their analysis. Since the majority of $\mathrm{Na}$ on the planet's limb at pressures $\lesssim \frac{1}{2} \mathrm{mbar}$ is ionized by stellar UV flux, the possible effects of neutral $\mathrm{Na}$ non-LTE level populations found by Barman et al. (2002) are reduced. In addition, since the $T-P$ profile we derive is cooler, a region where $\mathrm{Na}$ LTE level populations may not hold will be pushed out to lower pressure.

For our $T-P$ profile, we find an opaque forsterite cloud base at a pressure of several millibars. Predicting the exact location of the cloud base and vertical distribution of cloud material is challenging, but the $\mathrm{Na} \mathrm{D}$ feature is quite sensitive to these factors. An atmosphere incorporating the forsterite cloud at 1.8 mbar and ionization gives an $\mathrm{Na} \mathrm{D}$ feature less than observed (because of clouds, not ionization), but for a cloud base of at $5.4 \mathrm{mbar}$, we obtain a feature depth that matches observations with or without ionization.

More advanced modeling of photochemistry may reveal other nonequilibrium compounds, perhaps including a photochemical haze, that could both increase the importance of particulate absorption and decrease the extent to which the atmosphere is ionized. The potassium feature at $770 \mathrm{~nm}$ should be more sensitive to ionization than is $\mathrm{Na}$, because the former's ionization energy is $0.8 \mathrm{eV}$ less than that of $\mathrm{Na}$. We are currently updating our UV opacity database and collecting all available collisional parameters. Once this is completed, it will be worthwhile to revisit the sodium ionization problem, taking into account many levels of $\mathrm{Na}$ I and computing consistent non-LTE level populations of sodium together with the radiation field. More advanced global circulation models for HD 209458b will be of great interest, as they will give a better indication of the temperatures expected on the limb of the planet. It is likely that observations will push modeling in new directions, and data obtained across many wavelengths, both from the ground and from space, including predicted $\mathrm{K}, \mathrm{H}_{2} \mathrm{O}$, and $\mathrm{CO}$ features, will help gauge the importance of processes at work in HD 209458b's atmosphere and decide between explanations of the $\mathrm{Na} \mathrm{D}$ feature depth.

The authors acknowledge the valuable insight gained through discussions with Don Hunten and John Plane and support from grants NAG 5-10760, NAG 5-10450, and NAG 5-10629.

\section{REFERENCES}

Barman, T. S., Hauschildt, P. H., Schweitzer, A., Stancil, P. C., Baron, E., \& Allard, F. 2002, ApJ, 569, L51

Brown, T. M. 2001, ApJ, 553, 1006

Brown, T. M., Charbonneau, D., Gilliland, R. L., Noyes, R. W., \& Burrows, A. 2001, ApJ, 552, 699

Burrows, A., Hubbard, W. B., Lunine, J. I., \& Liebert, J. 2001, Rev. Mod. Phys., 73, 719

Castor, J. I., Dykema, P. G., \& Klein, R. I. 1992, ApJ, 387, 561

Charbonneau, D., Brown, T. M., Latham, D. W., \& Mayor, M. 2000, ApJ, 529, L45

Charbonneau, D., Brown, T. M., Noyes, R. W., \& Gilliland, R. L. 2002, ApJ, 568, 377

Cooper, C. S., Sudarsky, D., Milsom, J. A., Lunine, J. I., \& Burrows, A. 2003, ApJ, 586, 1320

Cunto, W., Mendoza, C., Ochsenbein, F., \& Zeippen, C. J. 1993, A\&A, 275, L5

Gudzenko, L. I., Evstigneev, V. V., Filippov, S. S., \& Yakovlenko, S. I.

1974, Teplofizika Vysokikh Temperatur, 12, 964
Guillot, T., \& Showman, A. P. 2002, A\&A, 385, 156

Henry, G. W., Marcy, G. W., Butler, R. P., \& Vogt, S. S. 2000, ApJ, 529, L41

Hubbard, W. B., Fortney, J. J., Lunine, J. I., Burrows, A., Sudarsky, D., \& Pinto, P. 2001, ApJ, 560, 413

Hubeny, I., \& Lanz, T. 1995, ApJ, 439, 875

Lee, L. C., Wang, X., \& Suto, M. 1987, J. Chem. Phys., 86, 4353

Mazeh, T., et al. 2000, ApJ, 532, L55

Mihalas, D. 1978, Stellar Atmospheres (2d ed.; San Francisco: Freeman)

Richardson, L. J., Deming, D., Wiedemann, G., Goukenleuque, C., Steyert, D., Harrington, J., \& Esposito, L. W. 2003, ApJ, 584, 1053

Seager, S., \& Sasselov, D. D. 2000, ApJ, 537, 916

Showman, A. P., \& Guillot, T. 2002, A\&A, 385, 166

Sudarsky, D., Burrows, A., \& Hubeny, I. 2003, ApJ, 588, 1121

Sudarsky, D., Burrows, A., \& Pinto, P. 2000, ApJ, 538, 885

Verner, D. A., \& Ferland, G. J. 1996, ApJS, 103, 467

Yoshino, K., Esmond, J. R., Parkinson, W. H., Ito, K., \& Matsui, T. 1996, Chem. Phys., 211, 387 (erratum 215, 429 [1997]) 